\title{
Handeln, Kritik, Verständigung
}

\author{
Max Weber - was Kommunikationswissenschaft und \\ Journalismus nach einem Jahrhundert von ihm lernen können
}

\section{Horst Pöttker}

Eingegangen: 1. Juni 2020 / Angenommen: 19. Juli 2020 / Online publiziert: 6. Oktober 2020 (C) Der/die Autor(en) 2020

Zusammenfassung Auch hundert Jahre nach Max Webers Tod können Kommunikationswissenschaft und Journalismus noch von ihm lernen: Sein grundlegender Begriff des sozialen Handelns stützt Legitimität und Chancen realistischer Medienkritik; sein Konzept einer großen Presse-Erhebung blickt umfassender auf öffentliche Kommunikationsprozesse als spätere Formeln und Modelle; seine Ethik-Vorstellungen fördern eine zivilisierte Grundhaltung des Abwägens in Politik, Wissenschaft und Journalismus; seine auf Rationalitätsbegriffe fixierte Typologie von Handlungsweisen regt zu einer Erweiterung an, die Grundprobleme sozialer Selbstregulierung besser erkennbar werden und u. a. auch durch nachhaltige Medienkommunikation lösbar erscheinen lässt; und sein Konzept einer verstehenden Soziologie weist ein halbes Jahrhundert vor Habermas auf die praktische Bedeutung des Erkenntnisinteresses an Verständigung hin, dem historisch-hermeneutische Disziplinen zu dienen haben. Die Argumentation verfolgt die These, dass der Wert eines Klassikers wie Max Weber vor allem darin liegt, bei fortschreitender Ausdifferenzierung und Parzellierung auch der (Kommunikations-)Wissenschaft selbst den Blick für gesellschaftliche Zusammenhänge von Medienphänomenen nicht zu verlieren.

Schlïsselwörter Handlungstheorie · Kommunikationsprozess · Medienkritik · Berufsethik $\cdot$ Folgenreflexivität $\cdot$ Verständigung $\cdot$ Max Weber

Dieser Aufsatz schreibt eine Abhandlung (Pöttker 2015) fort, die anlässlich der beiden Bände Siegfried Weischenbergs über Max Weber (Weischenberg 2012, 2014) entstanden ist.

Prof. i.R. Dr. H. Pöttker $(\bowtie)$

Institut für Journalistik, Technische Universität Dortmund, Emil-Figge-Str. 50, 44227 Dortmund, Deutschland

E-Mail: horst.poettker@tu-dortmund.de 


\section{Action, criticism, understanding}

Max Weber-what communication studies and journalism can still learn from him after a century

Abstract Even a hundred years after Max Weber's death, communication studies and journalism can still learn from him: his fundamental notion of social action supports the legitimacy and chances of realistic media criticism; his concept of a large press survey looks more comprehensively at public communication processes than later formulas and models; his ideas on ethics promote a civilized basic attitude of weighing up in politics, science and journalism; his typology of modes of action, which is based on concepts of rationality, encourages an expansion, which makes the basic problems of social self-regulation more easily recognizable and, among other things, allows for a better understanding of the social potential of the media; and his concept of an understanding sociology half a century before Habermas points to the practical significance of the cognitive interest in understanding, which historicalhermeneutic disciplines have to serve. The argumentation is based on the thesis that the value of a classic such as Max Weber lies above all in the fact that his work, with ongoing differentiation and parcelling out of even the (communication) sciences themselves does not lose sight of the social connections of media phenomena.

Keywords Action theory - Communication process - Media criticism · Professional ethics $\cdot$ Consequence reflexivity $\cdot$ Understanding $\cdot$ Max Weber

Niklas Luhmann hat gemeint, der Rückgriff auf Klassiker sei einer Innovationsträgheit in den Sozialwissenschaften geschuldet und blockiere den Mut, theoretische Innovationen zu wagen (vgl. Luhmann 1984, S. 7-8). Luhmann ging es darum, seiner Theorie sozialer Systeme die Aura von etwas völlig Neuem zu verschaffen. Er ist dann selbst in die Galerie der sozialwissenschaftlichen Klassiker eingerückt. Würde er seinen Adeptinnen und Adepten heute raten, die Systemtheorie zu vergessen, um Neues zu wagen?

Wichtig scheinen mir Klassiker immer noch, schon weil sie am Anfang der Ausdifferenzierung auch der Sozialwissenschaften selbst standen. So hatten sie noch Überblick über gesellschaftliche Phänomene und sahen deren Zusammenhänge. Max Weber, so meine These, ist in dieser Hinsicht ein eindrucksvolles Beispiel.

Über dem Folgenden steht die Frage, worin sich Kommunikationswissenschaft und Journalismus von Weber inspirieren lassen können, auch und gerade heute. Dabei geht es wieder einmal vor allem um das kritische Potential seines Werks (vgl. Hufnagel 1971).

Anlass gibt ein Gedenktag: Am 14. Juni 1920, vor 100 Jahren, ist Max Weber in München an einer Lungenentzündung gestorben. $\mathrm{Ob}$ er sie sich, wie oft behauptet (vgl. Seitz 2020), infolge einer Infektion mit dem Influenza-Virus (,Spanische Grippe“) zugezogen hatte, deren drei Wellen zwischen 1918 und 1920 auf dem Globus an die 50Mio. Menschenleben vernichtet haben, ist unklar. Im Obduktionsbericht steht dazu nichts (vgl. Graf 2020). 


\section{Der Begriff des sozialen Handelns und sein medienkritisches Potential}

Wie viele Klassiker ${ }^{1}$ war auch Max Weber Universalist. Bindestrich-Soziologien und auch die Kommunikations- und Medienwissenschaften haben sich erst später ausdifferenziert. Bei Weber reicht die Skala der Gegenstände von den sozialen Gründen des Untergangs der antiken Kultur bis zur Wirtschaftsethik der Weltreligionen, von der Lage der ostelbischen Landarbeiter bis zur „Wertfreiheit“ und „Objektivität“ sozialwissenschaftlicher Erkenntnis (die Anführungszeichen stammen von ihm selbst) oder von den Stufen religiöser Weltablehnung bis zum modernen Pressewesen².

Um das kritische und analytische Potential von Webers Werk für Kommunikationswissenschaft und Journalismus auszuloten, genügt es nicht, diejenigen seiner Texte zu lesen, die sich explizit mit der Presse befassen. Der Begriff des sozialen Handelns hält sein weit verzweigtes Werk zusammen. Er ist der erste in der Reihe „Soziologischer Grundbegriffe“, deren Erläuterungen an der Spitze seines postum von Marianne Weber herausgegebenen Hauptwerks „Wirtschaft und Gesellschaft“ stehen. Diesen ersten Teil hat Max Weber noch selbst für den Druck redigiert:

„Handeln“ soll [...] ein menschliches Verhalten (einerlei ob äußeres oder innerliches Tun, Unterlassen oder Dulden) heißen, wenn und insofern als der oder die Handelnden mit ihm einen subjektiven Sinn verbinden. „Soziales“ Handeln aber soll ein solches Handeln heißen, welches seinem von dem oder den Handelnden gemeinten Sinn nach auf das Verhalten anderer bezogen wird und daran in seinem Ablauf orientiert ist. (Weber 1976a, S. 1)

Weber betont, dass es ihm um den subjektiv gemeinten Sinn Einzelner geht. Deshalb wird kritisiert, seine Handlungstheorie bringe eine individualistische Perspektive mit sich, aus der soziale, viele Menschen betreffende Phänomene unsichtbar bleiben müssten. Er schreibt am gleichen Ort aber auch:

Es lassen sich innerhalb des sozialen Handelns tatsächliche Regelmäßigkeiten beobachten, d.h. in einem typisch gleichartig gemeinten Sinn [...] bei zahlreichen Handelnden verbreitete Abläufe von Handeln. Mit diesen Typen des Ablaufs von Handeln befaßt sich die Soziologie. (Weber 1976a, S. 14) ${ }^{3}$

Regelmäßigkeiten von Handlungen, Handlungsweisen - und damit Erwartbarkeit von Verhalten, gesellschaftlicher Zusammenhalt (vgl. Popitz 1980) - werden durch soziale Gebilde wie Staat, Kirche oder Verband, aber auch Einrichtungen wie Ehe, Eigentum oder formalisierte Berufspflichten gesichert. Das sozialwissenschaftliche Interesse kann sich in zwei Richtungen entwickeln: einerseits in Richtung der Frage, wie solche „Institutionen“ zustande kommen, von Menschen hervorgebracht und

\footnotetext{
${ }^{1}$ Zu den Kriterien des hier zu Grunde gelegten Klassiker-Begriffs vgl. Pöttker (2019, S. 438-440).

2 Einen gedrängten Überblick über diese Vielfalt verschafft der von Johannes Winckelmann herausgegebene Band mit Texten Webers zu Soziologie, Weltgeschichte und Politik (Weber 1968a), in dem dessen Forschungsinteresse an der Presse allerdings nicht berücksichtigt ist.

3 Weber hat ausdrücklich auf das ,,ungeheure Mißverständnis“ hingewiesen, ,,als ob eine ,individualistische" Methode eine (in irgendeinem möglichen Sinn) individualistische Wertung bedeute" (Weber 1976a, S. 9).
} 
verändert werden („Externalisierung“, vgl. Berger und Luckmann 1969, S. 112); und andererseits in Richtung der Frage, wie sie wirken, von Menschen berücksichtigt und angeeignet werden („Internalisierung“, vgl. Berger und Luckmann 1969, S. 65, 139-140).

Max Weber hat in beide Richtungen geblickt, indem er die Substanz von Institutionen nicht als „dauerhafte Regelsysteme“ (Donges 2013), sondern als Bündel von Handlungsweisen bestimmte, denen subjektiver Sinn, Muster von Legitimitätsvorstellungen unterliegen:

Für die Soziologie besteht der Tatbestand ,Staat“ nicht notwendig nur oder gerade aus den rechtlich relevanten Bestandteilen. [...] Die Deutung des Handelns muß von der grundlegend wichtigen Tatsache Notiz nehmen: daß jene dem Alltagsdenken oder dem juristischen (oder anderem Fach-)Denken angehörigen Kollektivgebilde Vorstellungen von etwas teils Seiendem, teils Geltensollendem in den Köpfen realer Menschen [...] sind, an denen sich deren Handeln orientiert, und daß sie als solche eine [...] kausale Bedeutung für die Art des Ablaufs des Handelns der realen Menschen haben. Vor allem als Vorstellungen von etwas Gelten- (oder auch: Nicht-Gelten-)Sollendem. (Weber 1976a, S. 6-7)

Damit behält Weber die objektive und die subjektive Seite der von Menschen sowohl hervorgebrachten als auch angeeigneten gesellschaftlichen Gegebenheiten zusammen im Auge ${ }^{4}$. Schon das macht ihn zum sozialwissenschaftlichen Klassiker.

Indem er Institutionen als Muster subjektiver Vorstellungen begreift, hält Weber die Denkmöglichkeit offen, dass an sozialer Ordnung auch anachronistisches Denken, kollektiver Irrtum, falsches Bewusstsein ${ }^{5}$ beteiligt sein kann:

Es verhüllen vorgeschobene „Motive“ und „Verdrängungen“ [...] oft genug dem Handelnden selbst den wirklichen Zusammenhang der Ausrichtung seines Handelns derart, daß auch subjektiv aufrichtige Selbstzeugnisse nur relativen Wert haben. In diesem Fall steht die Soziologie vor der Aufgabe, diesen Zusammenhang zu ermitteln und deutend festzustellen. (Weber 1976a, S. 4)

Wie bei Karl Marx die Kluft zwischen dem „Sein“ der sozio-ökonomischen Verhältnisse und dem falschen „Bewusstsein“ davon (vgl. Marx 1971), ist auch in Max Webers Begrifflichkeit die Unterscheidung subjektiver Vorstellungen von objektiven Gegebenheiten präsent. Auch Weber hält - allerdings ohne revolutionäre Perspektive - die Idee wach, dass Institutionen durch kritische Ermittlungen und Deutungen verändert werden können (vgl. z. B. Weber 1968b, S. 121), indem er die ihnen zu Grunde liegenden Muster „subjektiv gemeinten Sinns“ als entscheidende Faktoren betrachtet, um soziale Verhältnisse und Prozesse „erklärend verstehen“ zu können.

\footnotetext{
${ }^{4}$ Er hat darauf nicht ausdrücklich Anspruch erhoben, das blieb ein halbes Jahrhundert später Peter L. Berger und Thomas Luckmann vorbehalten, in deren viel zitiertem Buch Weber nur in einer Fußnote erwähnt wird (Berger und Luckmann 1969, S. 64). Sie propagieren: „Gesellschaft ist ein menschliches Produkt. Gesellschaft ist eine objektive Wirklichkeit. Der Mensch ist ein gesellschaftliches Produkt. Daß eine Analyse der gesellschaftlichen Welt, welche irgendeines dieser drei Elemente außer acht ließe, verzerrt wäre, dürfte [...] deutlich geworden sein." (S. 65).

5 Hier wie stets bei Weber im weitesten Sinne gemeint, also inklusive ,unterbewusster“ Muster subjektiver Sinngebung.
} 
Das ist für Kommunikationswissenschaft und Journalistik relevant, aber auch für den Journalistenberuf selbst. Denn es hält die Möglichkeit offen, mediale Darstellungen am Maßstab von objektiven Gegebenheiten zu messen.

Ob eine reale Welt jenseits der durch menschliche Beobachtung und Kommunikation konstruierten existiert, ist erkenntnistheoretisch weder gesichert noch widerlegbar. Menschen beobachten und kommunizieren aber nicht nur, wir essen und trinken, hungern, fürchten uns und leiden auch. Marx (vgl. 1971, S. 339), aber auch schon Lichtenberg in kritischen Aphorismen zum zeitgenössischen Konstruktivisten Kant (vgl. Lichtenberg 1913, S. 43) und später angelsächsische Pragmatiker wie Charles S. Peirce, John Dewey oder Karl R. Popper schieben deshalb die nicht entscheidbare erkenntnistheoretische Frage beiseite. Stattdessen unterscheiden sie in praktischer Absicht zwischen objektivem Sein und subjektivem Bewusstsein, um durch Kritik an und Korrektur von unzutreffender Beobachtung und Kommunikation besser mit der Welt zurechtzukommen: eine Position, um die der Journalistenberuf nicht herumkommt, wenn seine Verpflichtung zu zutreffender Information nicht aufgegeben wird.

Vor einem halben Jahrhundert dominierte in der Kommunikationswissenschaft ein von Marx inspirierter ideologiekritischer Blick, der mediale Darstellungen an der sozialen Wirklichkeit maß und auf ablenkende Verzerrungen hinwies.

In emanzipatorischen Forschungstraditionen ist diese Sichtweise noch zu finden. Im Mainstream der Medienfächer aber hat sich der ideologiekritische Impuls mit dem Siegeszug einer Denkweise verflüchtigt, die Realität nur als kommunikativ Konstruiertes zu Kenntnis nimmt und sich daher für Widersprüche zwischen Sein und Bewusstsein wenig interessiert.

Winfried Schulz hat diesen Wandel in die Metapher des Paradigmenwechsels vom geo- zum heliozentrischen Weltbild gefasst (vgl. Schulz 1989). Er hat dabei auf das Dilemma hingewiesen, dass erkenntnistheoretisch begründete Ignoranz gegenüber einer realen Welt, mit der Menschen zurechtkommen müssen, auch der Wissenschaft die Berechtigung zu fundierter Medienkritik nehmen kann:

Wenn das, was wir für Realität halten, ein soziales Konstrukt ist, das in starkem Maße von den Massenmedien bestimmt wird, müssen wir dann nicht die Vorstellung einer objektiven, unabhängig vom Beobachter existierenden Welt aufgeben - und damit auch wichtige Regeln der journalistischen Profession wie Sachlichkeit, Wahrhaftigkeit, Unparteilichkeit? (Schulz 1989, S. 145)

Schulz weist auf die erwähnte praktische Lösung hin:

In der Praxis kommt es darauf an, daß die Wirklichkeitskonstrukte als plausibel anerkannt werden und als Handlungsbasis taugen. [...] Eine solche pragmatische Sichtweise schließt die Möglichkeit ein, daß in einer Gesellschaft auch „falsche“ Vorstellungen von Wirklichkeit akzeptiert und zur Grundlage des kollektiven Handelns gemacht werden. (Schulz 1989, S. 143)

Das Kriterium der Anerkennung von Plausibilität bleibt allerdings nicht nur erkenntnistheoretisch, sondern auch gesellschaftspraktisch unbefriedigend, weil auch irreführende, ideologische, z. B. rassistische Wirklichkeitskonstrukte unter Umstän- 
den von vielen Menschen als ,wahr“" akzeptiert werden ${ }^{6}$. Das Problem ist offenbar unlösbar, ein echtes Dilemma. Bei Schulz kommt das u. a. durch den Verzicht darauf zum Ausdruck, ,berühmte Gründerväter der Kommunikationsforschung [...] als ,ptolemäisch“ oder ,kopernikanisch“ einzuordnen“" (Schulz 1989, S. 146).

Max Weber kann als Gründervater gelten, an dem sich zeigt, dass der Streit zwischen Positivismus (vgl. Adorno et al. 1969) und Konstruktivismus als theoretisch unnötiges wissenschaftspolitisches Konstrukt betrachtet werden kann. In seiner Handlungstheorie verbindet sich das Forschungsinteresse an der empirisch feststellbaren Gegebenheit sozialer Gebilde mit der Skepsis gegenüber der Realitätstüchtigkeit und Legitimität der ihnen zu Grunde liegenden Mentalitäten. Das ermöglicht sowohl das (journalistische) Gebot zur Objektivität im Sinne eines regulativen Ideals ${ }^{7}$ als auch den Gedanken an die Veränderbarkeit von Institutionen.

Ein Beispiel ist die Forschung zu Nachrichtenwertfaktoren. Aus deren empirisch „wertfrei“ festgestellten Katalogen werden in der Medienpraxis unter der Hand nicht selten normative Vorgaben für journalistisches Handeln (vgl. Donges 2013). Im Rahmen von Max Webers Handlungstheorie wäre der ursprünglich von Galtung u.a. intendierte medienkritische Impuls (vgl. Galtung und Ruge 1965; Östgaard 1965) noch legitim. Denn Webers konstitutiver Begriff des „subjektiv gemeinten Sinns“ schließt nicht aus, dass der journalistischen Orientierung an antizipierten Aufmerksamkeitspräferenzen des Publikums ein unrichtiges oder schädliches Bewusstsein zu Grunde liegt.

\section{Die Presse-Enquête und ihr medienanalytisches Potential}

Die kommunikationswissenschaftliche Bedeutung des Konzepts für eine umfassende Presse-Enquête, das Max Weber 1910 auf dem ersten Deutschen Soziologentag vorgestellt hat (Weber 1911), ist leichter erkennbar (vgl. Kutsch 1988; Weischenberg 2012, S. 78-164). Er konnte das Projekt zwar nicht realisieren, obwohl er bedeutende Zeitungswissenschaftler wie Karl Bücher daran beteiligen wollte. Kein Zweifel aber, dass der Entwurf wegen seines Reichtums an Fragen, Kriterien und methodischen Hinweisen immer noch eine wertvolle Hilfe bei Untersuchungsplanungen sein kann. In seiner Durchdringung des Forschungsfeldes in Tiefe und Breite liegt die zeitlose Bedeutung von Webers damaliger Konzeption.

Besonders aufschlussreich ist der ebenso kompakte wie reichhaltige „Vorbericht“, der erst ein Jahrhundert nach seiner Entstehung publiziert worden ist (vgl. Weischenberg 2012, S. 8-9). Um die Vielfalt der dort gestellten Fragen zu demonstrieren, genügt die Aufreihung der Faktoren, die Weber unter der für die handlungstheo-

\footnotetext{
${ }^{6}$ Ein Beispiel ist, dass die marxistische Geschichtsphilosophie in sozialistischen Gesellschaften selbst zu den ideologischen Vorstellungen gehört, die zur Grundlage kollektiven Handelns gemacht werden.

7 „Objektivität als ein abstraktes Ziel, als handlungsleitende Norm, als ein ,Ideal“. In dieser Betrachtungsweise hat Objektivität die Aufgabe, das faktische Verhalten der Journalisten zu leiten, die Medien anzuhalten, so genau und unparteilich wie möglich zu berichten. Man könnte auch, in Anlehnung an die Wissenschaftslogik des kritischen Rationalismus, von ,methodischer Objektivität“ sprechen." (Schulz 1989, S. 145) Kritischer Rationalismus als vermittelnde Position zwischen Positivismus und Konstruktivismus zeigt erwartbar Gemeinsamkeiten mit Webers erkenntnistheoretischer Konzeption.
} 
retische Basis charakteristischen Gliederungsrubrik „B. die Zeitungsgesinnung“ ins Auge fasst:

I. Die Produktion der Zeitungsgesinnung. Kollektivismus und Individualismus bei der Schaffung des Zeitungsinhalts. Die Anonymität der Zeitung: ihre Gründe: geschäftliche [...], politische [...], soziale [...] und kulturliche [...]. Ihre Wirkungen: auf den Journalisten, - auf die Förderung oder Hemmung der Erziehung der öffentlichen Meinung, - auf die politische und Kulturbedeutung der Zeitung als solcher. II. Die Beeinflussung der Gesinnung der Zeitung von aussen her. 1. Grad der Gebundenheit der Zeitung an ihre Tradition. [...] Die (allgemein oder in bestimmten Richtungen) formell programmgebundene Zeitung. [...] 3. Verhältnis der politischen Parteien zur formal „freien“ Presse. (Weber 2001a, S. 322-323)

Wie gedrängt und gleichzeitig detailliert Weber diese Untersuchungsgegenstände beschreibt, zeigt sich z. B. an seinen deutlich auf den gesellschaftlichen Zusammenhang bezogenen acht Erläuterungen zur „Produktion öffentlicher Meinung durch die Presse“. Davon nur eine als Beispiel:

4. Beeinflussung der Alltagssprache durch die Presse (wirkliches und angebliches Zeitungsdeutsch, bedingt durch Telegramm und Telephon) und Weiterwirken dieser Beeinflussung auf die Schrift- und Literatursprache (nur als sorgfältige philologische Facharbeit zuverlässig zu behandeln). Beeinflussung des Wissens- und Diskussionsbedürfnisses durch die zugleich „sachliche“ und ,emotionale" Stilisierung der Zeitungsnachrichten und der Zeitungskritik. Wirkliche und scheinbare Erweiterung des Horizonts, Anreicherung und Schematisierung des Denkens. (Nur Veranschaulichung an massenhaften konkreten Beispielen hat Wert.) (Weber 2001a, S. 323-324)

Eine längere Liste zum Gliederungsstichwort „A. das Zeitungsgeschäft“ ist vorangegangen (Weber 2001a, S. 317-322), und immer wieder soll die Presse-Enquête auch nach dem ,Zustand des Auslandes in dieser Hinsicht“ (Weber 2001a, S. 319) fragen.

Trotz dieser Fülle ist Webers Forschungskonzept kein gemischter Salat. Die Strukturierungskraft ergibt sich bereits aus dem Hinweis ,,auf die grossen Kulturprobleme der Gegenwart“, auf welche ,eine Erhebung über das Zeitungswesen [...] in letzter Linie ausgerichtet sein“ (Weber 2001a, S. 316) müsse:

I. Die Art der Bildung jenes Apparats von psychischen Suggestionsmitteln, durch welche die moderne Gesellschaft kontinuierlich den einzelnen sich einzufügen und anzupassen trachtet: die Presse als eins der Mittel zur Prägung der subjektiven Eigenart des modernen Menschen,

II. die durch die öffentliche Meinung, deren wichtigste Determinante heute die Zeitung ist, geschaffenen Bedingungen für die Entstehung, Erhaltung, Untergrabung, Umbildung von künstlerischen, wissenschaftlichen, ethischen, religiösen, politischen, sozialen, ökonomischen Kulturbestandteilen: Die Presse als Komponente der objektiven Eigenart der modernen Kultur. (Weber 2001a, S. 316) 
Auch hier zeigt sich: Weber beschränkt sein Denken weder auf die objektive noch auf die subjektive Seite der sozialen Realität. Wir Spätere glauben, uns zwischen Systemtheorie und Handlungstheorie entscheiden zu müssen; den Gründervätern der Sozialwissenschaften, auch Émile Durkheim oder Georg Simmel, war diese Alternative noch unbekannt.

Aus Webers Plänen für die Presse-Enquête lassen sich zahlreiche Forschungsfragen destillieren, von „Was bedeutet die privatwirtschaftliche Organisation der Presse für ihre Situation in der Gesellschaft im Allgemeinen und speziell in Hinblick auf Konzentrationsprozesse?“ über „Welche Macht haben die Medien, die öffentliche Meinung zu beeinflussen?“ bis zu ,Welche beruflichen Perspektiven haben die Journalisten?“ (Weischenberg 2014, S. 251) Man sieht: Weber sah Pressephänomene nicht isoliert, sondern im Kontext ihrer gesellschaftlichen Ursachen und Folgen. Aber statt auf dieses umfassende sozialwissenschaftliche Konzept griff die Kommunikationswissenschaft auch in Webers Heimat lange lieber auf spätere Strukturierungsmodelle aus den USA zurück, die Kommunikationsprozesse für sich beleuchten. Harold D. Lasswells bis heute benutzte, das Forschungsfeld höchst unvollständig erfassende Frage ,Wer sagt was auf welchem Kanal zu wem mit welcher Wirkung?" blickt von den Kommunikatoren aus über die Medieninhalte auf das Publikum und die sich dort zeigenden Wirkungen. Fragen nach dem „Warum“, den Absichten und Interessen von Kommunikatoren, oder nach den Rückwirkungen des Publikums, des kulturellen Kontextes auf die Handlungsweisen der Produzenten werden in der auf den Propagandaforscher Lasswell zurückgehenden Formel nicht gestellt. Probleme der Aussagenentstehung mussten in dieser Perspektive aus dem Fokus kommunikationswissenschaftlicher Aufmerksamkeit geraten (vgl. Weischenberg 2014, S. 131-132).

Diese Defizite sind heute weniger erkennbar, weil kulturanalytische und -vergleichende Ansätze zur Erklärung journalistischen Handelns an Bedeutung gewonnen haben (vgl. z. B. Hanitzsch 2007, 2009; Hanusch 2009). Gleichwohl sind Spuren davon noch immer zu finden. Eine grobe Themenanalyse der 75 Aufsätze, die in den Jahrgängen 2018 und 2019 in den Fachzeitschriften Medien \& Kommunikationswissenschaft und Publizistik erschienen sind, ergibt elf Texte (14,7\%), deren Fragestellungen sich erkennbar auf Strukturen der Entstehung von Medieninhalten beziehen; doppelt so viele $(28,0 \%)$ nehmen hauptsächlich Medieneinflüsse und Rezeptionseffekte (,Wirkungen“) in den Blick ${ }^{8}$.

Wenn kommunikationswissenschaftliche Forschung ihr Interesse auf Rezeptionsmechanismen konzentriert, lässt sie Fragen nach den Interessen von Kommunikatoren und ihren Organisationen liegen, die die Aussagenentstehung beeinflussen. Frank Böckelmann hat deshalb eine Reduktion der Kommunikationswissenschaft auf Wirkungsforschung kritisiert (vgl. Böckelmann 1975, S. 94-238). Er erkannte darin ein Ausweichen vor der kritischen Frage nach der Struktur der Massenkommunikation im Ganzen, die er - einem in den 1970er Jahren verbreiteten Paradigma entsprechend - als Verfallsprodukt bürgerlicher Öffentlichkeit in der spätkapitalistischen Gesellschaft verstand (vgl. Böckelmann 1975, S. 7-93). Anders als auf

\footnotetext{
${ }^{8}$ Die meisten anderen Beiträge konzentrieren sich auf kommunikationswissenschaftliche Selbstverständnisse, Methoden und Theorieentwürfe.
} 
Bourdieu oder den von ihm reichlich zitierten Habermas hat sich Böckelmann nicht auf Max Weber bezogen, wozu beigetragen haben mag, dass der ,Vorbericht“ zu der Zeit noch in Archiven schlummerte. Hätten er und andere sich an Weber erinnert, hätten sie vermeiden können, mit der verständlichen Kritik an der Engführung der Medienforschung gleich in einen anderen Gedankenkäfig zu geraten.

Journalismusforschung teilt zwar das Interesse an Rezeptionseffekten, weil das Ankommen von Informationen beim Publikum zur journalistischen Aufgabe gehört. Ebenso intensiv muss sie sich aber auch um Prozesse der Aussagenentstehung, z. B. redaktionsinterne Auswahl- und Transfermechanismen kümmern. Hier liegen die Probleme der selektiven Bereitstellung von Informationen, von denen die öffentliche Aufgabe des Journalistenberufs verlangt, dass sie nicht nur zutreffen (Richtigkeit), sondern auch im Interesse der Allgemeinheit liegen (Wichtigkeit).

Nach Webers Plan wäre z.B. zu untersuchen gewesen:

Von wem und was schweigt die Zeitung und aus was für, in ihrer Eigenart und den Bedürfen ihres Publikums liegenden, Gründen? (Weber 2001a, S. 324)

Abgesehen von medienkritischen Initiativen ${ }^{9}$ kümmert sich die Kommunikationsforschung um Lücken medial hergestellter Öffentlichkeit oder gar eine Systematik ihrer Gründe bisher kaum. Als Ursachen für solche Lücken kommen nicht nur Zensur und wirtschaftliche Zwänge, sondern z.B. auch Tabus und kulturelle Selbstverständlichkeiten, journalistische Routinen, volkspädagogische Neigungen von Journalistinnen und Journalisten, Überforderung von materiellen und intellektuellen Recherchekapazitäten oder die Verstopfung von Kommunikationskanälen mit Informationsmüll in Frage (vgl. Pöttker 2014).

Max Weber konnte medienkritische Fragen wie die nach dem (Ver-)Schweigen und seinen Gründen noch problemlos mit seiner Grundidee einer systematischen empirischen Wissenschaft verbinden. Er hat gemahnt,

dass, ehe man an solche Fragen geht, über die es sehr leicht ist, ein hübsches Feuilleton, über die es unglaublich schwer ist, eine wissenschaftliche Darstellung zu bieten, eine breite Unterlage von Erfahrungen und Analysen zu schaffen ist. (Weber 2001a, S. 324)

\section{Zwei Ethik-Begriffe und ihr berufspraktisches Potential}

Beruf soll jene Spezifizierung, Spezialisierung und Kombination von Leistungen einer Person heißen, welche für sie Grundlage einer kontinuierlichen Versorgungs- oder Erwerbschance ist. (Weber 1976b, S. 80)

\footnotetext{
9 Z.B. die „Initiative Nachrichtenaufklärung“ in Deutschland (http://www.derblindefleck.de/, zugegriffen 10. Mai 2020) oder das „Project Censored“ in den USA (https://www.projectcensored.org/, zugegriffen 10. Mai 2020).
} 
Die Einkommenschance ergibt sich daraus, dass die speziellen Leistungen, die einen Beruf ausmachen, bei anderen Menschen besondere Bedürfnisse erfüllen, was deren Bereitschaft bedingt, für diese Leistungen zu bezahlen. Das führt zu einem weiteren Begriff, den man in Webers berühmtem Aufsatz „Die protestantische Ethik und der Geist des Kapitalismus“ findet:

Nun ist unverkennbar, daß schon in dem deutschen Worte „Beruf“ ebenso wie in vielleicht noch deutlicherer Weise in dem englischen „,calling“, eine religiöse Vorstellung: - die von einer von Gott gestellten Aufgabe - wenigstens mitklingt. (Weber 1947, S. 63)

Berufe, so lassen sich die beiden Texte Webers verknüpfen, sind Bündel spezieller Leistungen, Kenntnisse und Fähigkeiten von Menschen, die ihnen die realistische Aussicht auf ein dauerhaftes Einkommen sichern, weil sich durch die Spezialisierung eine Aufgabe (ob von Gott gestellt, sei offengelassen) besonders effektiv und verlässlich erfüllen lässt, die für andere Menschen von Nutzen ist. Das dafür notwendige Bewusstsein von der beruflichen Aufgabe nennen wir Professionalität oder Berufsethos.

Ein Berufsethos überschneidet sich mit der allgemeinen Moral, deckt sich aber nicht mit ihr, weil die verlässliche Konzentration auf eine professionelle Aufgabe Anforderungen an das Handeln von Berufstätigen stellen kann, die nicht mit der Quintessenz der Moral, der Goldenen Regel (,Was du nicht willst, das man dir tu’, das füg' auch keinem andern zu!“"), übereinstimmen (vgl. Pöttker 2012).

In seinem ebenfalls berühmten, Anfang 1919 vor studentischem Publikum gehaltenen Vortrag ,Politik als Beruf“" hat sich Weber konkret und wohlwollend über den Journalistenberuf geäußert (vgl. Weber 2001b, S. 335). Bekannter als diese vor allem in der Journalistik beachtete Passage ist Webers Gegenüberstellung von Gesinnungsund Verantwortungsethik am Ende des Vortrags. Gesinnungsethik bedeutet, in einer Konfliktsituation ohne Rücksicht auf die konkreten Folgen des eigenen Tuns oder Lassens einem Prinzip, etwa einer beruflichen Aufgabe, treu zu bleiben; Verantwortungsethik dagegen beachtet die konkreten Auswirkungen des eigenen Handelns, um sie verantworten zu können.

Weber wird oft zustimmend Engagement für die Verantwortungsethik unterstellt. Das gibt sein Text jedoch nicht her, in dem es heißt, dass

Gesinnungsethik und Verantwortungsethik nicht absolute Gegensätze [sind], sondern Ergänzungen, die zusammen erst den echten Menschen ausmachen, den, der den „Beruf zu Politik“ haben kann. (Weber 2001b, S. 347)

Verantwortungsethik und Gesinnungsethik sollen sich Weber zufolge gegenseitig zügeln. Das erfordert bei widersprüchlichen Vorgaben Kompromisse. Dieses berufsethische Erfordernis dürfen wir auch auf den Journalismus beziehen, zumal Weber ihn für einen politischen Beruf hielt.

Ein aktuelles Beispiel ist die Ziffer 4 der Publizistischen Grundsätze des Deutschen Presserats (,Pressekodex“) ${ }^{10}$, die notwendige „Grenzen der Recherche“ regelt. Im Sinne der allgemeinen Moral erklärt sie verdeckte Recherche als eine Täuschung

10 https://www.presserat.de/pressekodex.html. 
des Gegenübers grundsätzlich für illegitim. Im Interesse der journalistischen Berufspflicht soll sie aber gerechtfertigt sein, ,wenn damit Informationen von besonderem öffentlichen Interesse beschafft werden, die auf andere Weise nicht zugänglich sind“.

Solche Kompromisse in Form von Ausnahmeregelungen sind für Berufsethiken charakteristisch. Sie erfordern im Einzelfall ein Abwägen zwischen der allgemeinen Moral einerseits, in diesem Fall der Verwerflichkeit von bewussten Irreführungen, und der professionellen Aufgabe andererseits, in diesem Fall der Pflicht, umfassende Transparenz herzustellen, gerade auch von verborgen gehaltenen Missständen.

Max Webers auf Abwägen hinauslaufendes Ethik-Konzept ist besonders für Journalistinnen und Journalisten in der Mediengesellschaft wichtig. Vieles geschieht hier nur, weil bestimmte Akteure wollen, dass über ihr Handeln berichtet wird. Das reicht von der Inszenierung öffentlicher Rituale für die TV-Kamera bis zu Amokläufen und politisch motivierten Terrorakten. Dürfen Journalistinnen und Journalisten (noch) Verantwortung für solche Folgen ihres beruflichen Handelns von sich weisen, indem sie sich gesinnungsethisch auf das traditionelle Selbstbild des unbeteiligten Beobachters berufen? Oder dürfen sie verantwortungsethisch wegen solcher (möglichen) Folgen auf Berichterstattung verzichten?

Eine Orientierung an Webers Ethik-Konzept kann eine in konkreten Konfliktfällen eigenständig abwägende Haltung fördern, die Journalistinnen und Journalisten je mehr brauchen, desto mehr ihr berufliches Handeln zur strukturellen Bedingung dessen wird, was in der Welt geschieht. Dazu gibt es im Pressekodex neuerdings einige Regeln. Jenseits des traditionellen Prinzips professioneller Distanz zum Geschehen halten sie dazu an, mögliche konkrete Folgen des Berichtens auf das Berichtete zu reflektieren; z.B.

\section{Richtlinie 8.11 - Opposition und Flucht}

Bei der Berichterstattung über Länder, in denen Opposition gegen die Regierung Gefahren für Leib und Leben bedeuten kann, ist zu bedenken: Durch die Nennung von Namen oder Fotoveröffentlichungen können Betroffene identifiziert und verfolgt werden. Auch kann die Veröffentlichung von Einzelheiten über Geflüchtete und ihre Flucht dazu führen, dass zurückgebliebene Verwandte und Freunde gefährdet oder noch bestehende Fluchtmöglichkeiten verbaut werden.

In der journalistischen Praxis kann selbstreflexives Abwägen Sensibilität dafür fördern, dass die eigene Berichterstattung allein schon durch die Inszenierung von Ereignissen von interessierter Seite instrumentalisiert werden kann.

Gesinnungsethik zeichnet sich u. a. durch beharrliches Festhalten an einmal formulierten Prinzipien und Werten aus. Sie orientiert sich daher gern an traditionellen, aus der Vergangenheit übernommenen Regeln. In der Zeit des digitalen Umbruchs, in der auch berufsethische Regularien des Journalismus sich wandeln (müssen), gewinnt Webers auf abwägende Eigenverantwortlichkeit setzendes und daher individuelle Freiheitsräume schützendes (vgl. Marty 2019) Ethik-Konzept besondere Bedeutung.

Allerdings: Wie sollen Journalistinnen und Journalisten beurteilen, ob und ggf. wie die eigene Anwesenheit eine Situation gravierend verändert? Zügelung von Gesinnungs- durch Verantwortungsethik ist in hochkomplexen Gesellschaften vor 
das Problem gestellt, dass dort Konsequenzen von Handlungen für die Akteure oft nicht absehbar sind, weil sie erst nach komplizierten Transformationsprozessen viel später wirksam werden. Zum Beispiel sind Klimaschäden durch CO2-Emissionen den kollektiv verursachenden Industrieunternehmen nur schwer zuzurechnen (vgl. E. Pöttker 2014). Für den individuellen Energieverbrauch gilt das noch mehr.

Wenn es aufgrund der Undurchschaubarkeit von Handlungsfolgen und fehlendem Verantwortungsbewusstsein zu einem charakteristischen Interaktionsmangel zwischen Personen(gruppen) und Institutionen kommt und soziale Selbstregulierungsmechanismen in der Folge versagen (vgl. Pöttker 1997, S. 124-191), ergibt sich für den Journalistenberuf eine ungewohnte Aufgabe: Journalistisch hergestellte Transparenz darf sich nicht mehr auf bereits geschehene Ereignisse beschränken, sondern sollte - im Rahmen eines pluralistischen Meinungsangebots - auch mögliche Auswirkungen diverser Handlungsweisen beim Warenkonsum, beim Wählen, beim Nutzen technischer Geräte usw. ins Auge fassen.

\section{Max Webers Handlungstheorie und eine mögliche Entwicklung ihres gesellschaftskritischen Potentials}

Der Begriff der auf subjektive und daher möglicherweise falsche Vorstellungen gegründeten (sozialen) Handlungsweisen behält sein kritisches Potential auch dann, wenn die auf ihn aufbauende Typologie Max Webers sich ungeachtet ihrer Geläufigkeit in Sozialwissenschaften und Alltagskultur nicht als alternativlos erweist.

Wie jedes Handeln kann auch das soziale Handeln bestimmt sein 1. zweckrational: durch Erwartungen des Verhaltens von Gegenständen der Außenwelt und von anderen Menschen und unter Benutzung dieser Erwartungen [...] als „Mittel“ für rational, als Erfolg erstrebte und abgewogene eigene Zwecke, 2. wertrational: durch bewußten Glauben an den [...] unbedingten Eigenwert eines bestimmten Sichverhaltens rein als solchen und unabhängig vom Erfolg, - 3. affektuell, insbesondere emotional [...], - 4. traditional: durch eingelebte Gewohnheit. (Weber 1976a, S. 12)

Bei Weber begründet diese Typologie die Anschauung, dass die Moderne von einer unaufhaltsamen Zunahme zweckrationaler, zwar effizienzsteigernder, aber disziplinierter, kalter, lebensferner Handlungsweisen durchdrungen sei. Er trat dem mit einer sein Werk kontnuierlich konnotierenden Melancholie gegenüber, die in persönlichem Charisma eine Alternative sah - auch, aber nicht nur den historisch überwundenen Kontrast (vgl. Weber 1976c).

In der Tradition solcher Melancholie hat es in den Sozialwissenschaften nicht an einflussreicher Literatur gemangelt, in der das „Unbehagen in der Modernität“ als unheilbares oder nur durch Rückfall hinter die Aufklärung therapierbares Leiden des Subjekts im „stahlharten Gehäuse“ eigendynamischer Strukturen erscheint (z. B. Berger et al. 1975; aber auch Horkheimer und Adorno 1969 oder Habermas 1981). Lässt sich im Rahmen von Webers Handlungstheorie seine Typologie der vier Handlungsweisen so verändern, dass eine schärfere Sicht sowohl auf die Pathologien der Moderne als auch auf realistische Therapiemöglichkeiten dafür geöffnet wird - und 
auch auf die Bedeutung von Medien in diesem Zusammenhang? Weber selbst hat vorausschauend in solche Überlegungen eingewilligt, indem er nicht einmal auf den Versuch Anspruch erhob, eine ,,irgendwie erschöpfende Klassifikation der Typen des Handelns zu geben“ (Weber 1976a, S. 13).

Es liegt nahe, vom Begriff des sozialen Handelns aus einmal nicht nach dem Inhalt des subjektiv gemeinten Sinns, sondern nach der Art und Weise zu fragen, wie das eigene Handeln im Bewusstsein von Akteuren auf das Verhalten anderer bezogen wird. Ein mögliches Kriterium ist, ob das Verhalten des oder der anderen - z. B. als Nachahmung eines Vorbilds oder als abwehrende Reaktion auf ein abschreckendes Beispiel - nur als gegebene Bedingung des eigenen Handelns oder auch als etwas angesehen wird, das durch das eigene Handeln verändert werden kann. Im ersten Fall bietet sich der Begriff der rezeptiven Handlungsweise an, im zweiten der der Folgenreflexivität. Interaktion, der ein entscheidendes Potential zur Selbstregulierung sozialer Verhältnisse innewohnt, kann auf dieser Grundlage als soziale Beziehung verstanden werden, in der beide (alle) Seiten folgenreflexiv handeln (vgl. Pöttker 1997, S. 73-100).

Ohne ihr weiter nachzugehen, deutet Weber diese Möglichkeit der Unterscheidung von Verhaltens- oder Handlungsweisen selber an. Bloße

Nachahmung fremden Handelns [...] [würde] begrifflich dann nicht spezifisch „soziales Handeln“ sein, wenn sie lediglich reaktiv, ohne sinnhafte Orientierung des eigenen an dem fremden Handeln, erfolgt. [...] Wird dagegen z. B. fremdes Handeln nachgeahmt, weil es „Mode“ ist, als traditional, mustergültig oder als ständisch ,vornehm“ gilt, oder aus ähnlichen Gründen, so liegt die Sinnbezogenheit - entweder: auf das Verhalten der Nachgeahmten, oder: Dritter, oder: beider - vor. Dazwischen liegen [...] Uebergänge. (Weber 1976a, S. 11-12)

Dem Menschen als einem Wesen, das seine Welt selbst herstellen muss, wohnt naturgemäß ein fundamentales Bedürfnis nach Handlungsfolgen inne. In der modernen Gesellschaft wird dieses Verlangen auch Weber zufolge permanent zurückgewiesen, weil der „Kosmos“ der kapitalistischen Wirtschaftsordnung in Verbindung mit allgegenwärtiger Bürokratie ,,als faktisch unabänderliches Gehäuse“ (Weber 1947, S. 37) auf seine Eigengesetzlichkeit pocht. Zwischen Institutionen moderner Gesellschaften und ihrem Publikum herrscht daher ein notorisches Defizit an Folgenreflexivität, Interaktion und Selbstregulierung. Eine therapeutische Rolle, die Medien und Journalismus dabei zufallen kann, ist wie erwähnt die Aufklärung über zeitversetzte Folgen von Alltagshandlungen, etwa im Bereich von Umweltschutz und Klimawandel.

Nicht nur verlangt diese Rolle dem traditionellen Selbstverständnis des Journalismus Veränderungen ab. Für Journalistik und Kommunikationsforschung ergeben sich auch bei der Diagnose der Probleme neue Fragen. Medien können Selbstregulierungsdefizite verfestigen, die aus der institutionalisierten Zurückweisung von Folgenreflexivität herrühren, indem sie - etwa durch Gewinnspiele u. ä. - pseudosoziale Handlungsfolgen vortäuschen und so das Interaktionsbedürfnis einfrieren (vgl. Pöttker 1997, S. 228-256).

In Alltagssprache wie Medienpraxis und -forschung sind ungenaue Begriffe von „Interaktivität“" verbreitet (vgl. Neuberger 2010). Geht es, wo immer dieses Stichwort 
fällt, tatsächlich um Interaktion, d.h. um wechselseitige Kommunikation, die bei den Beteiligten von der Reflexion möglicher Folgen ihrer Handlungen begleitet wird? Bringt die Kommunikation in digitalen Netzen tatsächlich die Stärkung von realistischer Folgenreflexivität mit sich?

Solche besonders am Anfang der Digitalisierung verbreiteten Utopien lassen die produktiven Funktionen der herkömmlichen Massenmedien in der Wahrnehmung gesellschaftlicher und fachlicher Öffentlichkeiten verblassen. Wenn die Baumstruktur der Massenmedien (,one to many“) nur als zu überwindende Einengung von Kommunikationsfreiheit und -vielfalt gesehen wird, gerät in Vergessenheit, dass sie sich besser als die digitale Netzstruktur zur Bündelung von richtigen Informationen und wichtigen Argumenten zu einer Öffentlichkeit eignet, die ohne Überforderung der menschlichen Verarbeitungskapazität Verständigung und gesellschaftlichen Zusammenhalt fördert.

Auf digitalen Plattformen werden Kommunikationsinhalte und -stile allgemein zugänglich, die vor der Digitalisierung aufgrund der technologisch begrenzten Reichweite privater Kommunikation in Kleingruppen wie Familien oder Stammtischen keine öffentlichen Konsequenzen hatten, die zu verantworten gewesen wären. In welchem Maße ein darauf rückführbarer Verzicht auf Folgenreflexivität sich in der digitalen Kommunikation fortsetzt, wäre zu untersuchen. Wenn sich herausstellen sollte, dass es sich bei digitaler Netzkommunikation selten tatsächlich um Interaktion handelt, wäre auch das Selbstregulierungspotential digitaler Kommunikationsplattformen kritisch unter die Lupe zu nehmen.

Ein Erbe Max Webers bleibt dabei, dass auch auf solchen bisher wenig beackerten Forschungsfeldern systematisch-empirisch nach Mustern subjektiver Motivationen einschließlich möglicher Täuschungen zu fragen ist, die Kommunikatoren oder Rezipienten ihrem Medienhandeln unterlegen.

\section{Die verstehende Soziologie und ihr wissenschaftskritisches Potential}

Max Weber nennt sein Wissenschaftskonzept ,verstehende Soziologie“ und distanziert sich damit einerseits von ,dogmatischen“ Disziplinen wie „Jurisprudenz, Logik, Ethik, Ästhetik“. Denn mit „Sinn“ wird von ihm „,nicht etwa irgendein objektiv ,richtiger" oder ein metaphysisch ergründeter, wahrer" Sinn“ gemeint, sondern ,der tatsächlich [...] von dem oder den [...] Handelnden subjektiv gemeinte Sinn.“ (Weber 1976a, S. 1) Andererseits grenzt er seine ,deutende Soziologie“ (Weber 1976a, S. 7) aber auch von den Naturwissenschaften ab. Sie sei

bei „,sozialen Gebilden“ (im Gegensatz zu „Organismen“) in der Lage: über die bloße Feststellung von funktionellen Zusammenhängen und Regeln (,,Gesetzen“) hinaus etwas aller „Naturwissenschaft“ [...] ewig Unzugängliches zu leisten: eben das „Verstehen“ des Verhaltens der beteiligten Einzelnen [...]. Diese Mehrleistung der deutenden gegenüber der beobachtenden Erklärung ist freilich durch den wesentlich hypothetischeren und fragmentarischeren Charakter der durch Deutung zu gewinnenden Ergebnisse erkauft. Aber dennoch: sie ist gerade das dem soziologischen Erkennen Spezifische. (Weber 1976a, S. 7) 
Worin besteht dieses Besondere deutender Wissenschaft? Ist es von sozialer Bedeutung?

Webers Konzept der verstehenden Soziologie birgt ein wissenschaftskritisches Potential, das seine Relevanz z.B. erweist, wenn man die Entwicklung der letzten Jahrzehnte betrachtet. Es erschließt sich, wenn man sich an die (Ideal-)Typologie der Fachkulturen erinnert, die Jürgen Habermas 1965 in seiner Frankfurter Antrittsvorlesung (Habermas 1969a) entwickelt hat.

Das Fundament dieser begrifflichen Architektur legt Habermas, indem er von drei grundlegenden gesellschaftlichen Interessen an Wissenschaft ausgeht: einem technischen Erkenntnisinteresse an Verfügbarkeit von Prozessen, das zuverlässige Prognosen verlangt und für das sich das Modell der Naturwissenschaften eignet; einem praktischen Interesse an kommunikativer Verständigung, das zu erfüllen den „historisch-hermeneutischen“ Disziplinen obliegt; und einem emanzipatorischen Interesse an Befreiung aus „der Abhängigkeit von hypostasierten Gewalten“ (Habermas 1969a, S. 162) durch Selbstreflexion, das er der Philosophie und anderen kritischen Handlungswissenschaften zuschreibt.

Betrachtet man die Entwicklung der Wissenschaftskultur, zeigt sich ein scheinbar unaufhaltsames Wachsen und Gedeihen des naturwissenschaftlich-technischen Modells, dessen Hegemonie Habermas schon damals kritisiert hat (vgl. Habermas 1969 b, c). Auch emanzipatorische Interessen kommen in den Sozialwissenschaften bis heute zum Zuge, wenn man an die Bedeutung von Forschungsfeldern wie Migration, Armut oder Geschlechtergerechtigkeit denkt. Im Vergleich dazu ist die historisch-hermeneutische Arbeitsweise und mit ihr das praktische Erkenntnisinteresse an gesellschaftlicher Verständigung - einmal abgesehen von den Schutzräumen der Theologien und der Rechtswissenschaft - sowohl finanz- als auch aufmerksamkeitsökonomisch ins Hintertreffen geraten. Auch die Kommunikations- und Medienfächer betrachten die Abkehr von ihrer geisteswissenschaftlichen Tradition als Fortschritt und orientieren sich zunehmend am ,exakten“ Paradigma ${ }^{11}$, obwohl sie nicht nach Naturgesetzen suchen, sondern menschengemachte und daher zeitlich und kulturell differenzierte Gegenstände erklären und verstehen wollen.

Sich an einen Klassiker wie Weber zu halten, kann ein - schwaches - Mittel sein, einer Entwicklung entgegenzuwirken, in der das Erkenntnisinteresse an Verständigung vernachlässigt wird. Klassiker haben ihre grundlegenden Ideen (noch) in einer Weise formuliert, die an die ganze Öffentlichkeit und nicht nur an wissenschaftsimmanente Qualifikationshierarchien gerichtet war. Verständigung setzt Verstehen voraus, und das wiederum Verständlichkeit. Weber taugt, was z. B. Einfachheit und Verständlichkeit des Satzbaus betrifft (vgl. Langer et al. 2015), etwa im Vergleich zu Georg Simmel gewiss nicht als leuchtendes Vorbild. Aber das Ziel, mit seinen Analysen zur Verständigung über gesellschaftliche Verhältnisse beizutragen und so

\footnotetext{
11 Nur ein Anzeichen dafür: In den 1960er Jahren gehörte es in der Publizistik zum Standard, am Anfang von Studien die Geschichte des untersuchten Phänomens wenigstens zu skizzieren. Heute sind stattdessen Zahlen und Tabellen obligatorisch.
} 
an deren Gestaltung mitzuwirken ${ }^{12}$, durchzieht sein ganzes Werk. Sein Hinweis, dass mit dem Verstehen von einzelnen Handlungen (durch Geschichtswissenschaft) oder Handlungsweisen (durch verstehende Soziologie) (vgl. Weber 1976a, S. 9) eine der Naturwissenschaft unerreichbare Erkenntnisleistung vollbracht wird, betont die Unverzichtbarkeit der von Habermas ,historisch-hermeneutisch“ genannten Disziplinen für Verständigung und sozialen Zusammenhang. Missverständlich wäre dabei, rationales Verstehen mit Einverständnis oder gar Identifikation zu verwechseln:

Die volle „Nacherlebbarkeit“ ist für die Evidenz des Verstehens wichtig, nicht aber absolute Bedingung der Sinndeutung. [Manche] letzten ,Zwecke“ und „Werte“, an denen das Handeln eines Menschen erfahrungsgemäß orientiert sein kann, vermögen wir oft nicht voll evident zu verstehen, sondern unter Umständen zwar intellektuell zu erfassen, dabei aber [...], je radikaler sie von unseren eigenen letzten Werten abweichen, desto schwieriger uns durch die einfühlende Phantasie nacherlebend verständlich zu machen. Je nach Lage des Falles müssen wir dann uns begnügen, sie nur intellektuell zu deuten. (Weber 1976a, S. 2)

Wissenschaftspolitikerinnen und -manager, die historisch-hermeneutische Disziplinen - vielleicht nur heimlich - für Luxus und ihre Denkweisen für überholt, wenn nicht für ,unwissenschaftlich“ halten und gleichzeitig über Gefährdungen von gesellschaftlichem Zusammenhalt klagen, sollten sich an die eigene Nase fassen. Auch auf das legitimierende Argument, in solchen Fächern ginge es normativ und nicht ,wertfrei“ zu, hat Weber eine Antwort gegeben. Er hat betont, dass Wertvorstellungen, die die Auswahl von Untersuchungsobjekten und darauf gerichtete Fragestellungen prägen, unvermeidlich sind. Nach ihm haben die Offenlegung grundlegender Wertaxiome sowie Wertungsdiskussionen auch in einer empirischen Wirklichkeitswissenschaft, ,weit entfernt davon [...], ,sinnlos` zu sein, [...] ihren sehr erheblichen Sinn“ (Weber 1968c, S. 276).

Verstehen ohne Zwang zum Konsens, gegründet auf skeptischen Realismus und vernünftige Wertorientierungen, begleitet von selbstreflexivem Abwägen und weitblickendem Verantwortungsbewusstsein: Komponenten eines aus dem Werk Max Webers zu erschließenden methodologischen Konzepts, das auch in hochkomplexen Gesellschaften der Gegenwart mit ihrer tiefen sozialen und kulturellen Differenziertheit für Kommunikationswissenschaft und Journalismus noch beachtenswert ist.

Funding Open Access funding enabled and organized by Projekt DEAL.

Open Access Dieser Artikel wird unter der Creative Commons Namensnennung 4.0 International Lizenz veröffentlicht, welche die Nutzung, Vervielfältigung, Bearbeitung, Verbreitung und Wiedergabe in jeglichem Medium und Format erlaubt, sofern Sie den/die ursprünglichen Autor(en) und die Quelle ord-

12 Weber hat sich oft zu politischen Fragen geäußert, auch in journalistischen Medien wie der Frankfurter Zeitung (vgl. Weber 1980a). U.a. hat er sich Ende 1918 zu den Beratungen über die Verfassung der Weimarer Republik hinzuziehen lassen (vgl. Weber 1980b). Er gilt als einer ihrer „Väter“ neben Hugo Preuß und Friedrich Naumann. 
nungsgemäß nennen, einen Link zur Creative Commons Lizenz beifügen und angeben, ob Änderungen vorgenommen wurden.

Die in diesem Artikel enthaltenen Bilder und sonstiges Drittmaterial unterliegen ebenfalls der genannten Creative Commons Lizenz, sofern sich aus der Abbildungslegende nichts anderes ergibt. Sofern das betreffende Material nicht unter der genannten Creative Commons Lizenz steht und die betreffende Handlung nicht nach gesetzlichen Vorschriften erlaubt ist, ist für die oben aufgeführten Weiterverwendungen des Materials die Einwilligung des jeweiligen Rechteinhabers einzuholen.

Weitere Details zur Lizenz entnehmen Sie bitte der Lizenzinformation auf http://creativecommons.org/ licenses/by/4.0/deed.de.

\section{Literatur}

Adorno, T. W., Dahrendorf, R., Pilot, H., Albert, H., Habermas, J., \& Popper, K. R. (1969). Der Positivismusstreit in der deutschen Soziologie. Neuwied: Luchterhand.

Berger, P.L., \& Luckmann, T. (1969). Die gesellschaftliche Konstruktion der Wirklichkeit. Eine Theorie der Wissenssoziologie. M. e. Einl. v. Helmuth Plessner. Frankfurt a. M.: S. Fischer.

Berger, P. L., Berger, B., \& Kellner, H. (1975). Das Unbehagen in der Modernität. Frankfurt a. M.: Campus.

Böckelmann, F. (1975). Theorie der Massenkommunikation. Das System hergestellter Öffentlichkeit, Wirkungsforschung und gesellschaftliche Kommunikationsverhältnisse (2. Aufl.). Frankfurt a. M.: Suhrkamp.

Donges, P. (2013). Institutionen. In G. Bentele, H.-B. Brosius \& O. Jarren (Hrsg.), Lexikon Kommunikations- und Medienwissenschaft (2. Aufl.) (S. 128). Wiesbaden: Springer VS.

Galtung, J., \& Ruge, M. B. (1965). The structure of foreign news. Journal of Peace Research, 2, 64-91.

Graf, F.W. (2020). Mythen und Wahrheit: Weder Cato noch Plato. Über den Tod Max Webers am 14. Juni 1920 in München ranken sich Legenden. Zeit für eine Klarstellung. Süddeutsche Zeitung, 18. 6. 2020. https://www.sueddeutsche.de/kultur/mythen-und-wahrheit-weder-cato-noch-plato- 1. 4938721. Zugegriffen: 6. Aug. 2020.

Habermas, J. (1969a). Erkenntnis und Interesse. In J. Habermas (Hrsg.), Technik und Wissenschaft als „Ideologie“ (2. Aufl.) (S. 146-168). Frankfurt a. M.: Suhrkamp.

Habermas, J. (1969b). Technik und Wissenschaft als „Ideologie“ (2. Aufl.). Frankfurt a. M.: Suhrkamp.

Habermas, J. (1969c). Analytische Wissenschaftstheorie und Dialektik. Ein Nachtrag zur Kontroverse zwischen Popper und Adorno. In T.W. Adorno, R. Dahrendorf, H. Pilot, H. Albert, J. Habermas \& K. R. Popper (Hrsg.), Der Positivismusstreit in der deutschen Soziologie (S. 155-191). Neuwied: Luchterhand.

Habermas, J. (1981). Theorie des kommunikativen Handelns. 2 Bde. Frankfurt a. M.: Suhrkamp.

Hanitzsch, T. (2007). Deconstructing journalism culture: towards a universal theory. Communication Theory, 17, 367-385.

Hanitzsch, T. (2009). Zur Wahrnehmung von Einflüssen im Journalismus. Komparative Befunde aus 17 Ländern. Medien \& Kommunikationswissenschaft, 57, 153-173.

Hanusch, F. (2009). A product of their culture: using a value systems approach to understand the work practices of journalists. International Communication Gazette, 71, 613-626.

Horkheimer, M., \& Adorno, T.W. (1969). Dialektik der Aufklärung. Frankfurt a. M.: S. Fischer. (zuerst 1944)

Hufnagel, G. (1971). Kritik als Beruf. Der kritische Gehalt im Werk Max Webers. Frankfurt a. M.: Propyläen.

Kutsch, A. (1988). Max Webers Anregung zur empirischen Journalismusforschung. Die „Zeitungs-Enquête" und eine Redakteurs-Umfrage. Publizistik, 33, 5-31.

Langer, I., Schulz von Thun, F., \& Tausch, R. (2015). Sich verständlich ausdrücken (10. Aufl.). München: Ernst Reinhardt.

Lichtenberg, G. C. (1913). Aphorismen. Hrsg. v. Albert Leitzmann. Leipzig: Insel.

Luhmann, N. (1984). Soziale Systeme. Grundriß einer allgemeinen Theorie. Frankfurt a. M.: Suhrkamp.

Marty, C. (2019). Max Weber. Ein Denker der Freiheit. Weinheim: Beltz Juventa.

Marx, K. (1971). Die deutsche Ideologie (1845/46). In S. Landshut (Hrsg.), K. Marx, Die Frühschriften (S. 339-485). Stuttgart: Kröner.

Neuberger, C. (2010). Illusionäre Interaktion. In T. Eberwein \& D. Müller (Hrsg.), Journalismus und Öffentlichkeit: Eine Profession und ihr gesellschaftlicher Auftrag (S. 97-105). Wiesbaden: VS. 
Östgaard, E. (1965). Factors influencing the flow of news. Journal of Peace Research, 2, 39-63.

Popitz, H. (1980). Die normative Konstruktion von Gesellschaft. Tübingen: J.C.B. Mohr (Paul Siebeck).

Pöttker, E. (2014). Klimahaftungsrecht. Die Haftung für die Emission von Treibhausgasen in Deutschland und den Vereinigten Staaten von Amerika. Tübingen: Mohr Siebeck.

Pöttker, H. (1997). Entfremdung und Illusion. Soziales Handeln in der Moderne. Tübingen: Mohr Siebeck.

Pöttker, H. (2012). Öffentlichkeit und Moral. Zu Kernproblemen journalistischer Berufsethik. In M. Zichy, J. Ostheimer \& H. Grimm (Hrsg.), Was ist ein moralisches Problem? Zur Frage des Gegenstandes angewandter Ethik (S. 268-292). Freiburg: Karl Alber.

Pöttker, H. (2014). Geheim, verdrängt, unbekannt. Lücken von Öffentlichkeit: Worüber Medien gern schweigen - und warum sie das tun. medien \& zeit, 29(2), 13-30.

Pöttker, H. (2015). Max Weber als Klassiker der Journalistik und Kommunikationswissenschaft. Rezensionen:kommunikation:medien (r:k:m). Köln: Herbert von Halem. https://www.rkm-journal.de/ archives/18278. Zugegriffen: 31. März 2020.

Pöttker, H. (2019). „Die Presse verrät ihren Beruf“. Theodor Geiger (1891-1952) - ein (fast) vergessener Klassiker auch der Kommunikationswissenschaft. Medien \& Kommunikationswissenschaft, 67, 437-458.

Schulz, W. (1989). Massenmedien und Realität. Die „ptolemäische“ und die „,kopernikanische“ Auffassung. In M. Kaase \& W. Schulz (Hrsg.), Massenkommunikation. Theorien, Methoden, Befunde (S. 135-149). Opladen: Westdeutscher Verlag.

Seitz, N. (2020). Zum 100. Todestag Max Webers. Der Jahrhundertsoziologe und die Politik. Deutschlandfunk (DLF), 13. 6. 2020. https://www.deutschlandfunk.de/zum-100-todestag-max-webers-derjahrhundertsoziologe-und.724.de.html?dram:article_id=478576. Zugegriffen: 6. Aug. 2020.

Weber, M. (1911). Geschäftsbericht. In Deutsche Gesellschaft für Soziologie (Hrsg.), Verhandlungen des Ersten Deutschen Soziologentages vom 19. bis 22. Oktober 1910 in Frankfurt a. M. Reden und Vorträge (S. 39-62). Tübingen: J. C. B. Mohr (Paul Siebeck).

Weber, M. (1947). Die protestantische Ethik und der Geist des Kapitalismus. In M. Weber, Gesammelte Aufsätze zur Religionssoziologie (4. Aufl.) (S. 17-206). Tübingen: J.C.B. Mohr (Paul Siebeck).

Weber, M. (1968a). Soziologie - Weltgeschichtliche Analysen - Politik, Hrsg. von Johannes Winckelmann m. e. Einl. v. Eduard Baumgarten (4. Aufl.). Stuttgart: Kröner.

Weber, M. (1968b). Über einige Kategorien der verstehenden Soziologie. In J. Winckelmann (Hrsg.), M. Weber, Soziologie - Weltgeschichtliche Analysen - Politik. M. e. Einl. v. Eduard Baumgarten (4. Aufl.) (S. 97-150). Stuttgart: Kröner.

Weber, M. (1968c). Der Sinn der „Wertfreiheit“ der Sozialwissenschaften. In J. Winckelmann (Hrsg.), M. Weber, Soziologie - Weltgeschichtliche Analysen - Politik. M. e. Einl. v. Eduard Baumgarten (4. Aufl.) (S. 263-310). Stuttgart: Kröner.

Weber, M. (1976a). Soziologische Grundbegriffe. In J. Winckelmann (Hrsg.), M. Weber, Wirtschaft und Gesellschaft. Grundriss der verstehenden Soziologie (5. Aufl.) (Bd. 1, S. 1-30). Tübingen: J.C.B. Mohr (Paul Siebeck).

Weber, M. (1976b). Wirtschaft und Gesellschaft. Grundriss der verstehenden Soziologie. Hrsg. v. Johannes Winckelmann (5. Aufl.). Bd. 1. Tübingen: J.C.B. Mohr (Paul Siebeck).

Weber, M. (1976c). Die charismatische Herrschaft und ihre Umbildung. In J. Winckelmann (Hrsg.), M. Weber, Wirtschaft und Gesellschaft. Grundriss der verstehenden Soziologie (5. Aufl.) (Bd. 2, S. 654-687). Tübingen: J.C.B. Mohr (Paul Siebeck).

Weber, M. (1980a). Gesammelte Politische Schriften. Hrsg. v. J. Winckelmann (4. Aufl.). Tübingen: J.C.B. Mohr (Paul Siebeck).

Weber, M. (1980b). Parlament und Regierung im neugeordneten Deutschland. In J. Winckelmann (Hrsg.), M. Weber, Gesammelte Politische Schriften (4. Aufl.) (S. 306-406). Tübingen: J.C.B. Mohr (Paul Siebeck).

Weber, M. (2001a). Vorbericht über eine vorgeschlagene Erhebung über die Soziologie des Zeitungswesens. In H. Pöttker (Hrsg.), Öffentlichkeit als gesellschaftlicher Auftrag. Klassiker der Sozialwissenschaft über Journalismus und Medien (S. 316-325). Konstanz: UVK.

Weber, M. (2001b). Politik als Beruf. In H. Pöttker (Hrsg.), Öffentlichkeit als gesellschaftlicher Auftrag. Klassiker der Sozialwissenschaft über Journalismus und Medien (S. 329-347). Konstanz: UVK.

Weischenberg, S. (2012). Max Weber und die Entzauberung der Medienwelt. Theorien und Querelen eine andere Fachgeschichte. Wiesbaden: Springer VS.

Weischenberg, S. (2014). Max Weber und die Vermessung der Medienwelt. Empirie und Ethik des Journalismus - eine Spurenlese. Wiesbaden: Springer VS. 
Prof. i.R. Dr. Horst Pöttker war bis zur Pensionierung 2013 Professor für Theorie und Praxis des Journalismus an der (Technischen) Universität Dortmund, danach bis 2018 Seniorprofessor an der Universität Hamburg und ist heute dort und an der Technischen Universität Hamburg Lehrbeauftragter. 